Check for updates

Cite this: DOI: 10.1039/d0cp01258k

\title{
Density scaling of structure and dynamics of an ionic liquid $\dagger$
}

\author{
Henriette Wase Hansen, ${ }^{\text {abc }}$ Filippa Lundin, ${ }^{b}$ Karolina Adrjanowicz, (D) d \\ Bernhard Frick, (D) ${ }^{c}$ Aleksandar Matic (D) ${ }^{b}$ and Kristine Niss (D) ${ }^{a}$
}

\begin{abstract}
Room temperature ionic liquids are salts with low melting points achieved by employing bulky and asymmetrical ions. The molecular design leads to apolar and polar parts as well as the presence of competing Coulomb and van der Waals interactions giving rise to nano-scale structure, e.g. charge ordering. In this paper we address the question of how these nano-scale structures influence transport properties and dynamics on different timescales. We apply pressure and temperature as control parameters and investigate the structure factor, charge transport, microscopic alpha relaxation and phonon dynamics in the phase diagram of an ionic liquid. Including viscosity and self diffusion data from literature we find that all the dynamic and transport variables studied follow the same density scaling, i.e. they all depend on the scaling variable $\Gamma=\rho^{\gamma / T}$, with $\gamma=2.8$. The molecular nearest neighbor structure is found to follow a density scaling identical to that of the dynamics, while this is not the case for the charge ordering, indicating that the charge ordering has little influence on the investigated dynamics.
\end{abstract}

Received 5th March 2020 Accepted 31st May 2020

DOI: $10.1039 / d 0 c p 01258 k$

rsc.li/pccp the phase diagram can be envisaged for transport properties, although they do not a priori coincide with the isochrones. It has previously been shown that a density scaling of the viscosity or the relaxation time as a function of temperature and pressure applies for many molecular liquids and polymers, i.e. pressure and temperature behavior can be brought to collapse when expressed in terms of the parameter $\Gamma=\rho^{\gamma} / T$, where $\gamma$ is a material specific constant and $\rho$ is the density. ${ }^{3-6}$ If different transport and dynamical variables obey density scaling with identical value of $\gamma$, then they also have identical isolines in the phase diagram.

In ionic liquids the conductivity, $\sigma$, is a key transport property. Conductivity decreases rapidly with decreasing temperature or increasing pressure and isoconductivity lines can readily be identified in the $(T, P)$ phase diagram. In contrast to simple liquids, ionic liquids are structurally ordered on the nanometer scale due to the competition between Coulomb and van der Waals interactions. ${ }^{7}$ Despite this structuring, density scaling in ionic liquids has been shown to hold for both viscosity, ${ }^{8-11}$ conductivity ${ }^{11-16}$ and self diffusion..$^{11}$ Yet, density scaling has not been demonstrated before for the alpha relaxation in ionic liquids. Dielectric spectroscopy is the main technique for probing the alpha relaxation in nonconducting liquids, especially when it comes to high pressure studies. This technique, though, is not applicable for ionic liquids because the contribution to the dielectric signal from the conductivity overshadows the contribution of the alpha relaxation. However, the alpha relaxation can be accessed in the low viscosity liquid by quasi-elastic neutron spectroscopy (QENS).

In this work we investigate the pressure and temperature dependence of structure and dynamics with respect to density 


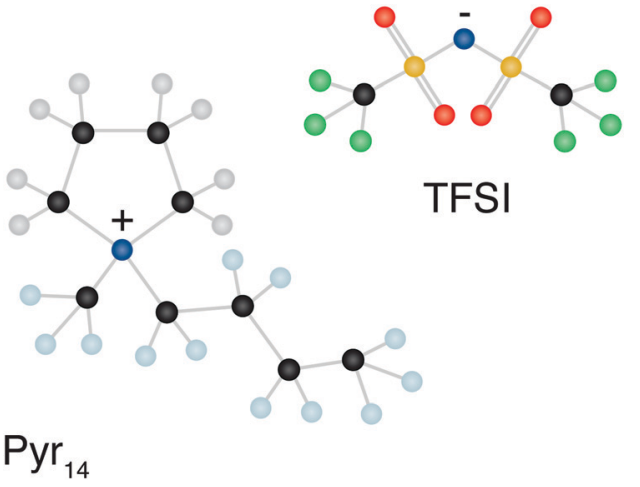

Fig. 1 Molecular structure of the sample studied in this work, the ionic liquid Pyr14TFSI (1-butyl-1-methylpyrrolidinium bis(trifluoromethanesulfonyl)imide). The methyl group and butyl chain on the cation are deuterated in the sample used for neutron scattering in order to mask the dynamics of these side groups. The anion has little incoherent scattering, thus mainly the cation dynamics is observed in the neutron data.

scaling in ionic liquids. We have studied the ionic liquid Pyr14TFSI (1-butyl-1-methylpyrrolidinium bis(trifluoromethanesulfonyl)imide). ${ }^{17,18}$ The molecular structure of the sample is shown in Fig. 1. The dynamics and structure are studied as a function of pressure and temperature with a range of techniques. This includes pressure and temperature dependent density, structure factor with X-ray diffraction, conductivity from dielectric spectroscopy and microscopic dynamics with neutron spectroscopy. This allows us to directly investigate how structure and dynamics are connected in the phase diagram and to extend our previous studies ${ }^{19,20}$ by testing density scaling of various properties also in an ionic liquid.

\section{Experiments}

To follow alpha relaxation dynamics in $(T, P)$ space we measure conductivity spectra and QENS simultaneously on the backscattering instrument IN16B at Institut Laue-Langevin (ILL) ${ }^{21}$ by use of the high-pressure cell developed for this type of experiment. ${ }^{22}$ In the neutron spectroscopy experiment the large incoherent scattering cross section of hydrogen gives access to the self-motion of a molecule by measuring directly the dynamic structure factor. By double Fourier transform in space and time, the measured dynamic structure factor transforms into the self-correlation function. We use a sample where both the methyl group and the butyl side chain on the cation are deuterated to mask internal cation dynamics. Thus the data is mainly sensitive to the self-motion dynamics of the carbon ring of the cation (Fig. S1 for more details, ESI $\uparrow$ ). The energy resolution of the IN16B instrument gives access to relaxation dynamics on nanosecond timescale, ${ }^{23}$ specifically the motion of the cation measured at $Q \sim 1 \AA^{-1}$, while the conductivity spectra reflect the macroscopic ion transport of cations and anions. Moreover, QENS spectra of Pyr14TFSI were measured on the time-of-flight instrument IN5 simultaneously with conductivity at the ILL. ${ }^{21}$ IN5 has a coarser energy resolution,

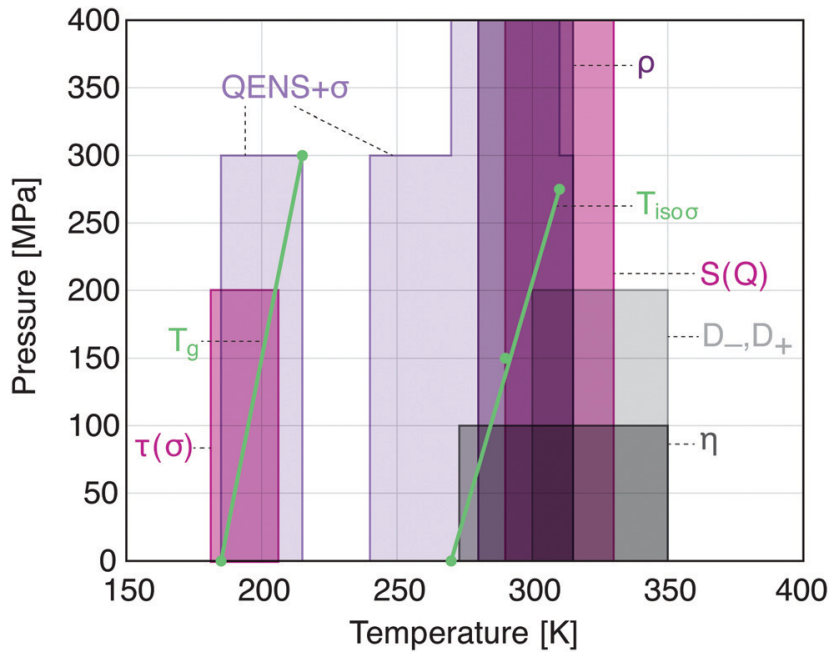

Fig. 2 Phase diagram illustrating the areas of the temperaure-pressure phase diagram we have explored with different experimental techniques. QENS $+\sigma$ : quasielastic neutron scattering and simultaneous conductivity, $\tau(\sigma)$ : conductivity peak, $\rho$ : density, $S(Q)$ : structure factor, $\eta$ : viscosity from ref. $27, D_{-}, D_{+}$: self diffusion from ref. $27 . T_{\text {iso, } \sigma}$ is the line along which the green data in Fig. 3 are collected. Data in Fig. 7(a) are taken at the endpoints of the $T_{\mathrm{g}}$-line.

in this case with an energy resolution of $0.1 \mathrm{meV}$ providing a window into picosecond dynamics.

All data shown from IN16B and IN5 have been reduced in LAMP $^{24}$ in the standard way. The data from IN16B are shown as a sum over $Q$ in the range from 0.5 to $1.8 \AA^{-1}$. The IN5 data have been summed over $Q$ in constant $Q$-slices, $Q=1.2$ to $1.8 \AA^{-1}$. The $Q$-resolved data do not change the conclusions regarding density scaling, which is the focus of this paper, but some examples are shown in the ESI, $\dagger$ (mentioned also in the text when relevant).

In addition to the conductivity measurements performed simultaneously with the neutron experiments, conductivity was also measured in a separate experiment at Silesia University along four isobars. From these data we report the conductivity relaxation time $\tau_{\sigma}$ (determined as described in ref. 14).

The structure factor was measured by using the P-jump cell ${ }^{25}$ at the small angle X-ray scattering beamline I22 at the Diamond synchrotron source. This was done as a function of pressure and temperature. The data was reduced in the standard way using the DAWN software. ${ }^{26}$

Density, $\rho$, has been measured as a function of pressure and temperature at Roskilde University, and interpolated and extrapolated using a fit with the Tait equation. More information about the density measurements can be found in the ESI. $\dagger$

The pressure and temperature range of the all the experiments are illustrated in Fig. 2.

\section{Results and analysis}

\subsection{Density scaling of dynamics}

In Fig. 3 we show three $(T, P)$ isoconductivity state points and the corresponding QENS spectra from IN16B along with data 

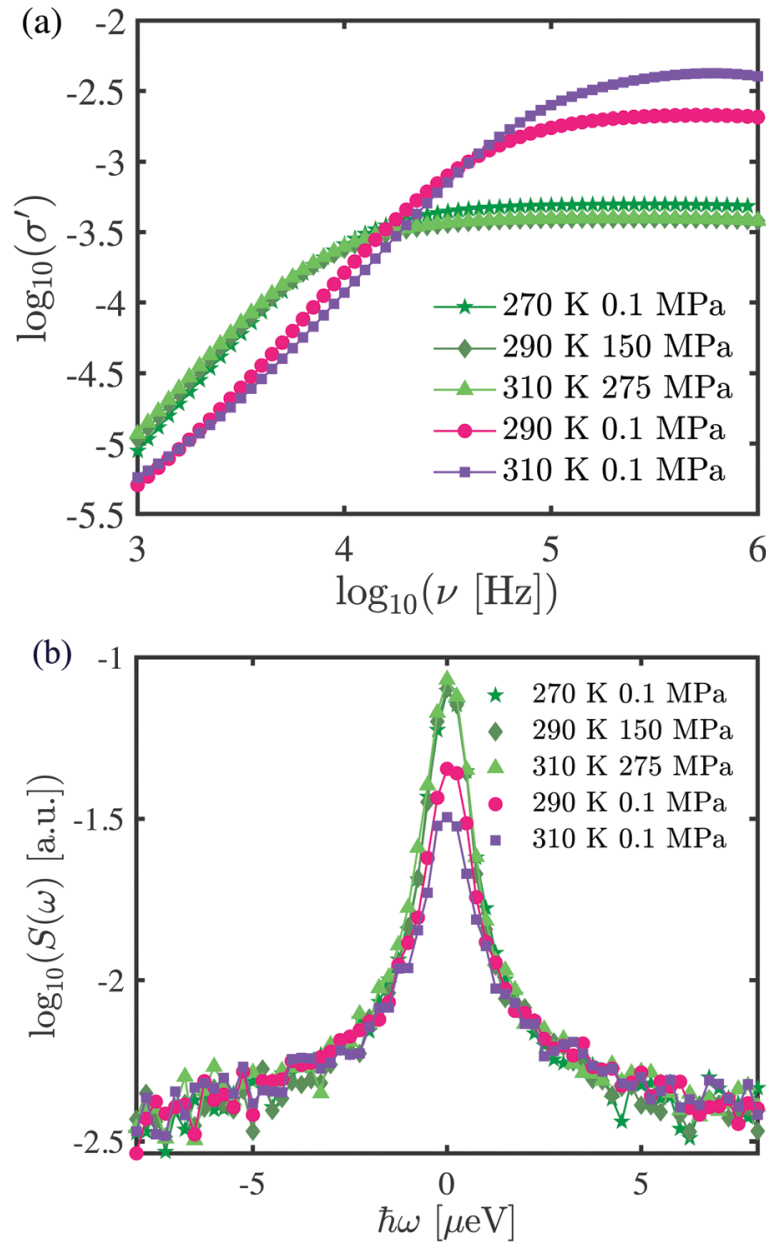

Fig. 3 Frequency dependent conductivity and QENS spectra from the simultaneous measurements at different pressures and temperatures. (a) Real part of the frequency dependent conductivity measured at different state points. Three isoconductivity state points are shown in green. (b) Neutron QENS spectra from IN16B summed over $Q$. The three isoconductivity state points are again shown in green.

from two other state points on the ambient pressure isobar for comparison. At ambient pressure, we observe the conductivity to increase with temperature as expected. In the QENS spectra, the elastic intensity goes down and the spectra broadens slightly as the temperature is increased reflecting increased dynamics. The broadening of the quasi-elastic signal is inversely proportional to the time scale of the alpha relaxation. For the three isoconductivity state-points, the three corresponding neutron spectra also collapse meaning that the alpha relaxation time is invariant at these state points. Thus by determining an isoconductivity line in the $(T, P)$ phase diagram, we have identified an isochrone of the alpha relaxation along which the microscopic dynamics is invariant. This observation leads us to the hypothesis that not just these two isolines, but isolines in a broader range of the phase diagram and of different dynamical properties collapse. As mentioned above, if different transport and dynamics variables obey density scaling with the same scaling variable $\Gamma=\rho^{\gamma} / T$ then they have identical isolines in the phase diagram. Thus, testing density scaling of different properties also tests the hypothesis of identical isolines.
Fig. 4(a) demonstrates density scaling of the alpha relaxation signal as probed by neutron scattering using elastic fixed window scans (EFWS) from IN16B ${ }^{23}$ summed over all momentum transfers $(Q)$ for statistical reasons along five isotherms and two isobars (note that no change in scaling is observed with $Q$ as shown in Fig. S2, ESI $\dagger$ ). The scaling exponent is determined by visual inspection as the value that gives the best collapse of data. The conductivity, simultaneously measured using the combined neutron and dielectric cell, is shown in Fig. 4(b) along with ambient pressure conductivity, $\sigma$, from ref. 27-29. In addition Fig. 4(b) shows the conductivity relaxation time $\tau_{\sigma}$ (determined as described in ref. 14) measured in a separate experiment along four isobars. The conductivity relaxation time gives an alternative measure of charge transport, which is readily determined in the viscous liquid where conductivity is strongly frequency dependent. The data in Fig. 4 is shown in absolute units, while theoretical arguments suggest that reduced units should be used..$^{30,31}$ We show the same data using reduced units in the ESI $\dagger$ (Fig. S3), but there is little difference in the case of Pyr14TFSI, which has also been shown in ref. 11 . The scaling exponent, $\gamma$, is in all cases determined by visual inspection. The uncertainty is approximately \pm 0.2 for the high temperature data but could be higher at low temperatures where density has been extrapolated. The density scaling has previously been reported in ref. 11 for two of the properties (diffusion and viscosity) and the exponent has been determined from the glass transition line in ref. 32. Both these earlier works find $\gamma=3$ for Pyr14TFSI. We consider this to be consistent with our result within the estimated error bars, but we find $\gamma=2.8$ gives a significantly better collapse of the EFWS data which are measured at high temperature were density is also measured, meaning that this is the range with highest certainty of the determined $\gamma$-value. In addition we also find that $\gamma=2.8$ gives a better collapse for the diffusion data from ref. 27 which were used in ref. 11. The density scaling of these data is shown in Fig. 4(c) using $\gamma=2.8$. We have shown all the density scaling figures in the ESI, $\dagger$ using $\gamma=3$ (Fig. S4, ESI $\dagger$ ).

From Fig. 4, it is clear that charge transport (conductivity and conductivity relaxation time), self diffusion, viscosity and the microscopic alpha relaxation all obey identical density scaling, i.e. a universal behavior is observed with the same exponent, $\gamma=2.8$, for all quantities scaling with $\Gamma=\rho^{\gamma} / T$. Identical density scaling does not imply that all properties behave in the same way. In fact, viscosity and diffusion have significantly different $\Gamma$-dependence in Fig. 4(c). However, identical density scaling does imply that the relative influence of density and temperature is the same for all properties. The consequence is that knowledge of the isolines in the phase diagram of one of these properties predicts the isolines of the others. In Table 4.2 of ref. 33 there is a compilation of the density scaling exponents from conductivity and viscosity for a number of different liquids. The exponents are very close, especially when considering ILs without hydrogen bonding, suggesting that this finding could be general.

The value of the scaling exponent, $\gamma=2.8$, found from the density scaling of our data agrees well with previous observations for ionic liquids with $\gamma=2-3$ reported in literature..$^{8,10,16,34}$ 
(a)
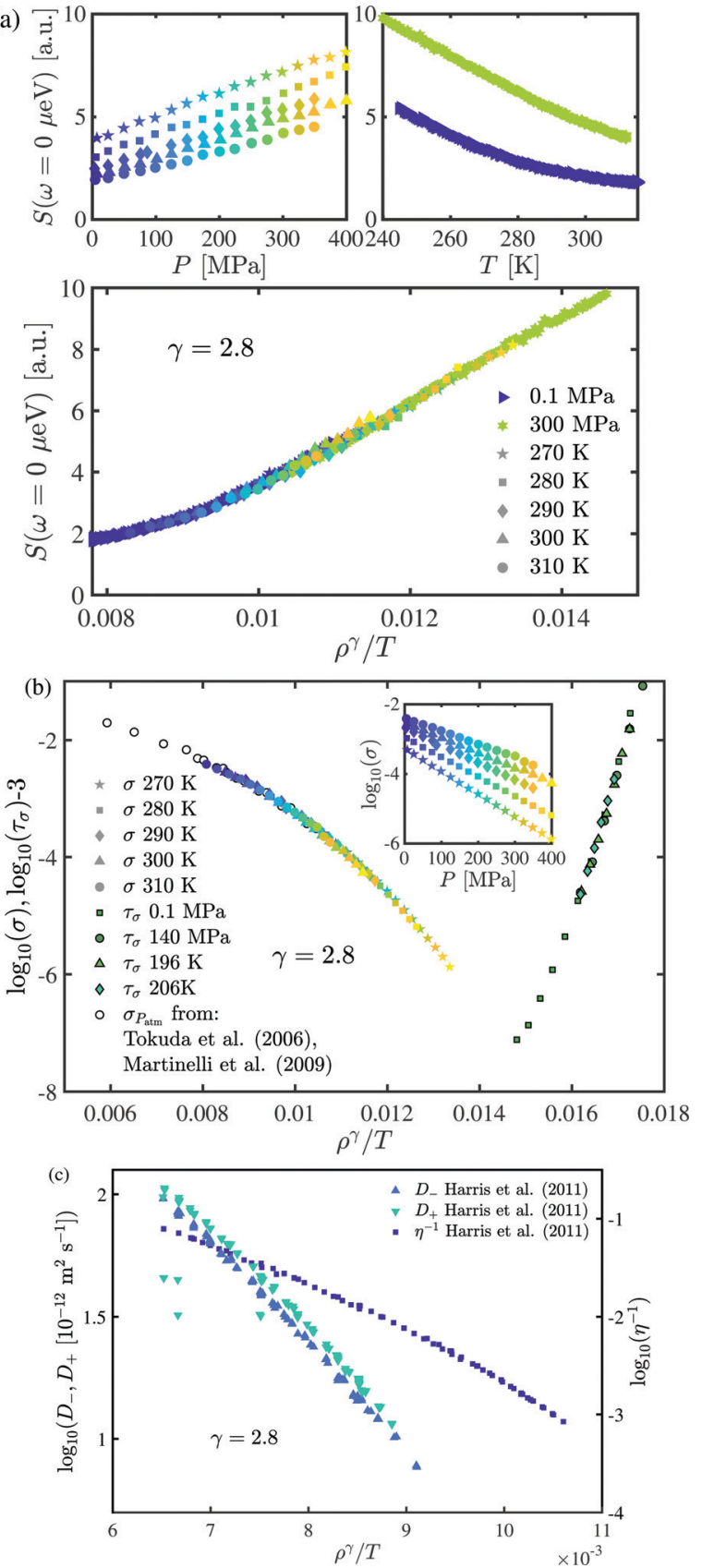

Fig. 4 Density scaling of dynamic and transport variables. The symbols used and shown in the legend of fig. (a) and (b) refer to the temperature of the measurement. The colors illustrate pressure with dark blue at ambient pressure and yellow at $400 \mathrm{MPa}$. (a) Elastic fixed window scans (EFWS) of Pyr14TFSI on five isotherms and two isobars from the neutron backscattering instrument IN16B summed over $Q$. The top panels of fig. (a) show the data measured on isotherms as a function of pressure and the data from the isobars as a function of temperature. The bottom part of fig. (a) shows all the EFWS data plotted as a function of the scaling variable $\Gamma=\rho^{\gamma} / T$. (b) Shows conductivity on five isotherms measured simultaneously with the neutron scattering data on IN16B. The inset shows the conductivity as a function of pressure, while conductivity is shown as a function of $\Gamma=\rho^{\gamma / T}$ in the main figure. The main part of figure (b) also shows conductivity relaxation time $\tau_{\sigma}$ measured at lower temperatures along four isobars and ambient pressure conductivity, $\sigma$, from ref. 28 and 29. (c) Shows inverse viscosity and diffusion constant of anion and cation from ref. 27.
It is worth noting that this places ionic liquids at an intermediate level between the typical values for simple van der Waals liquids, where $\gamma$ is often between 4 and 6 , and hydrogen bonded liquids where $\gamma$ typically is below 2 in the cases where density scaling holds for hydrogen bonding liquids. ${ }^{35-38}$

\subsection{Density scaling of structure}

Considering the presence of nano-scale ordering ${ }^{17,18}$ in ionic liquids it is of interest to connect the changes in the dynamics to the evolution of the structure.

In Fig. 5 we show the structure factor at four state points. The peak observed around $Q \sim 0.8 \AA^{-1}$, is related to charge ordering, i.e. the fact that the ions pack in a manner that insures charge neutrality on intermediate length scales $^{7}$ whereas the peak at $Q \sim 1.2 \AA^{-1}$ is the molecular peak arising from nearest neighbor distances, typically found also in ordinary molecular liquids. ${ }^{39}$ The inset of Fig. 5(a) illustrates the investigated state points chosen around an approximate isoconductivity line, such that the involved pressure and temperature changes have equivalent effect on the conductivity. In Fig. 5(a) the data are shown as measured, whereas in (b)-(d) the data are shown as a function of the reduced (dimensionless) wave vector $\tilde{Q}=Q \rho^{-1 / 3}$ to compensate for trivial density effects. Fig. 5(b) shows two isoconductivity state points, (c) shows two isothermal state points and (d) shows two isobaric state points. It is seen in Fig. 5 that the intensity of the charge ordering peak clearly depends on pressure, but only weakly depends on temperature. The intensity of the molecular peak, however, does not change dramatically with neither temperature nor with pressure. Yet, the collapse of the structure
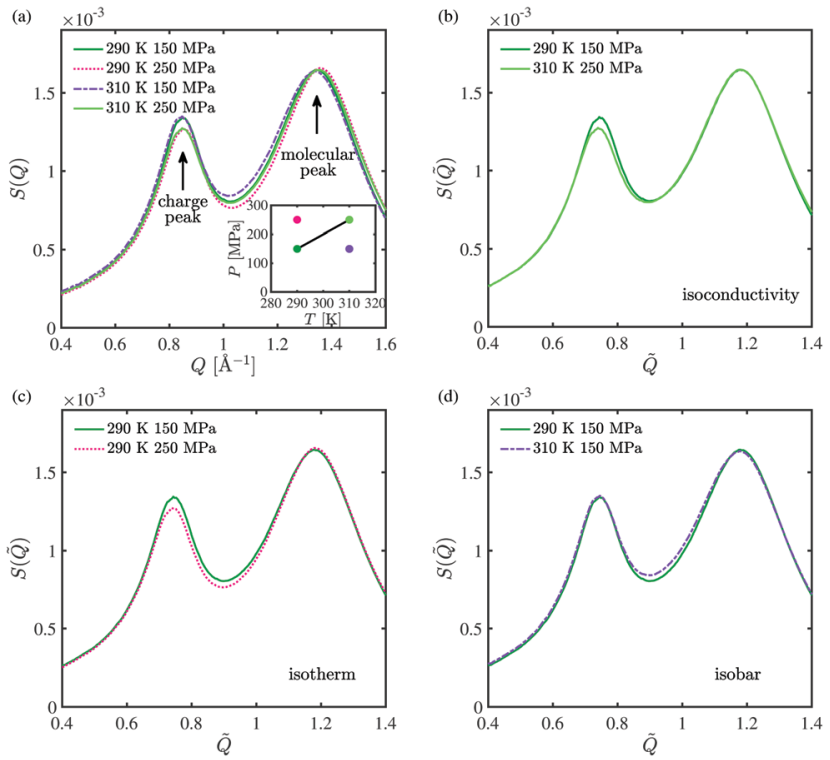

Fig. 5 X-ray structure factor from 122 on Pyr14TFSI. (a) Diffraction data from the four state point as measured, the inset shows the position in the face diagram. (b) -(d) shown as a function of $\tilde{Q}=Q \rho^{-1 / 3}$. (b) The two isoconductivity state points, (c) isotherm state points and (d) isobaric state points. The changes are subtle, but it is clear that the collapse of the molecular peak is very good for the isoconductivity points, while this is not the case for the charge peak. 
factors around the molecular peak of the two isoconductivity state points is better than along the isobar and the isotherm, obvious in particular in the minima between the two peaks.

To obtain a more quantitative comparison of the structure factors of the different state points we fit the peak positions of three isotherms measured from 0.1 to $400 \mathrm{MPa}$ and plot them as a function of $\Gamma$ (Fig. 6). This plot shows that the molecular peak positions collapse in a density scaling while the position of charge ordering peak does not (see also Fig. S5, ESI $\dagger$ ). This shows that the molecular peak position is identical along the isolines of conductivity, viscosity and microscopic alpha relaxation. The observed density scaling of the molecular peak, but

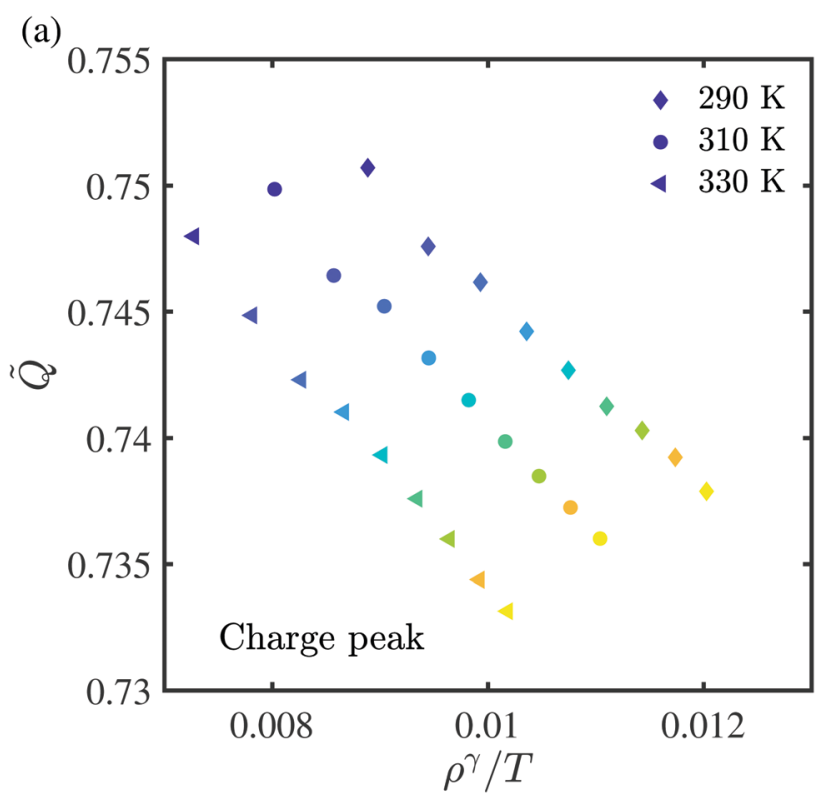

(b)

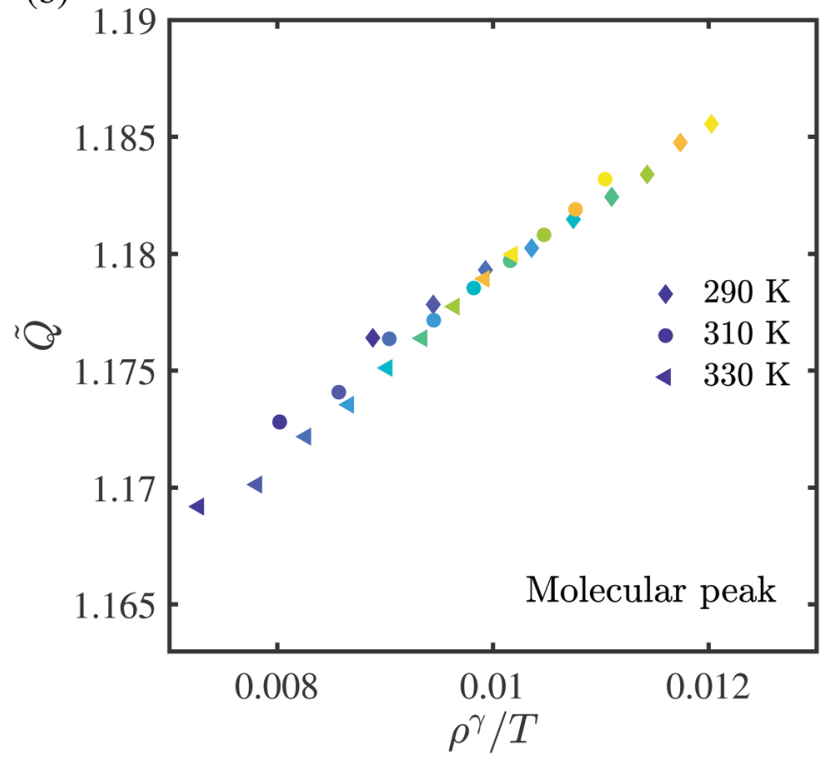

Fig. 6 Density scaling of the two peak positions shown for data measured on three isotherms at $290 \mathrm{~K}, 310 \mathrm{~K}$ and $330 \mathrm{~K}$ from 0.1 to $400 \mathrm{MPa}$ both the charge peak and the molecular peak are shown in terms of $\tilde{Q}$ and as a function of $\Gamma=\rho^{\gamma / T}$ with $\gamma=2.8$. (a) Charge peak. (b) Molecular peak. not of the charge ordering peak, indicates that the nearest neighbor order is directly connected to the dynamics, while the longer range charge order has a less direct effect on the dynamic variables.

\subsection{Boson peak behavior}

So far the analysis mainly focused on dynamics and structure in the low viscosity liquid phase, i.e. above the melting line. In the following we consider instead dynamics along the glass transition line. In a recent paper, ${ }^{32}$ Lima et al. show that the $T_{\mathrm{g}}(P)$ line of Pyr14TFSI is also a line of constant $\Gamma=\rho^{\gamma} / T$, in the pressure range considered in our work. The exponent $\gamma=3$ from that work is as discussed in Section 3.1 consistent with the value $\gamma=2.8$ we find. From frequency dependent conductivity measurements we find that $T_{\mathrm{g}}(P)$-line is also an isoline of the conductivity (Fig. S6, ESI $\dagger$ ). The $T_{\mathrm{g}}(P)$ line is by definition an isochrone of the macroscopic alpha relaxation and this is therefore another example of how different dynamic isolines collapse. Moreover, it shows that the $T_{\mathrm{g}}(P)$-line can be determined from conductivity measurements consistent with studies showing how the conductivity of series of chemically similar systems can be scaled when plotted as $T / T_{\mathrm{g}}{ }^{29}$

Along the glass transition line $T_{\mathrm{g}}(P)$, we have simultaneously with the frequency dependent conductivity measured QENS spectra of Pyr14TFSI on the time-of-flight instrument IN5 at the ILL. ${ }^{21}$ IN5 has a coarser energy resolution, in this case with an energy resolution of $0.1 \mathrm{meV}$ providing a window into picosecond dynamics, i.e. faster than what was probed with IN16B. The alpha relaxation is on the scale of minutes at $T_{\mathrm{g}}$ and therefore completely out of the experimental window of IN5. Instead the experiments probe vibrational dynamics, in particular, the excess in the density of states, a characteristic dynamical feature of glass-forming liquids, referred to as the Boson peak. ${ }^{40}$ Merged with the Boson peak is the fast relaxation which is seen as a broadening of the central peak. Fig. 7 shows the neutron spectra of Pyr14TFSI measured at the $T_{\mathrm{g}}(P)$-line and in the glassy state with the spectrometer IN5. As for the IN16B experiment, we have used a sample with deuterated methyl and butyl chains, which means that the data is mostly sensitive to the dynamics of the carbon ring of the cation. Fig. 7 also shows equivalent spectra from a van der Waals liquid 5-polyphenyl ether (PPE) and a hydrogen bonded liquid dipropylene glycol (DPG) for comparison all plotted as a function of a reduced energy unit following work by some of us in ref. 19. Data are summed over $Q$, as there is no observable difference in behavior for the different values of $Q$ (Fig. S7 for Pyr14TFSI, ESI $\dagger$ ).

For Pyr14TFSI, the Boson peak at $T_{\mathrm{g}}$ is observed to be a shoulder merged with the fast relaxations, resembling the van der Waals liquid (PPE), contrary to the hydrogen bonded liquid (DPG), where an actual peak is observed. However, for the Boson peak in the glass, there is no obvious qualitative difference between the three materials. This suggests that the difference at $T_{\mathrm{g}}$ stems from the fast relaxation, which is much more prominent in the van der Waals liquid and ionic liquid than in the hydrogen bonded liquid. The qualitative difference may relate to the proposed connection between fragility and 

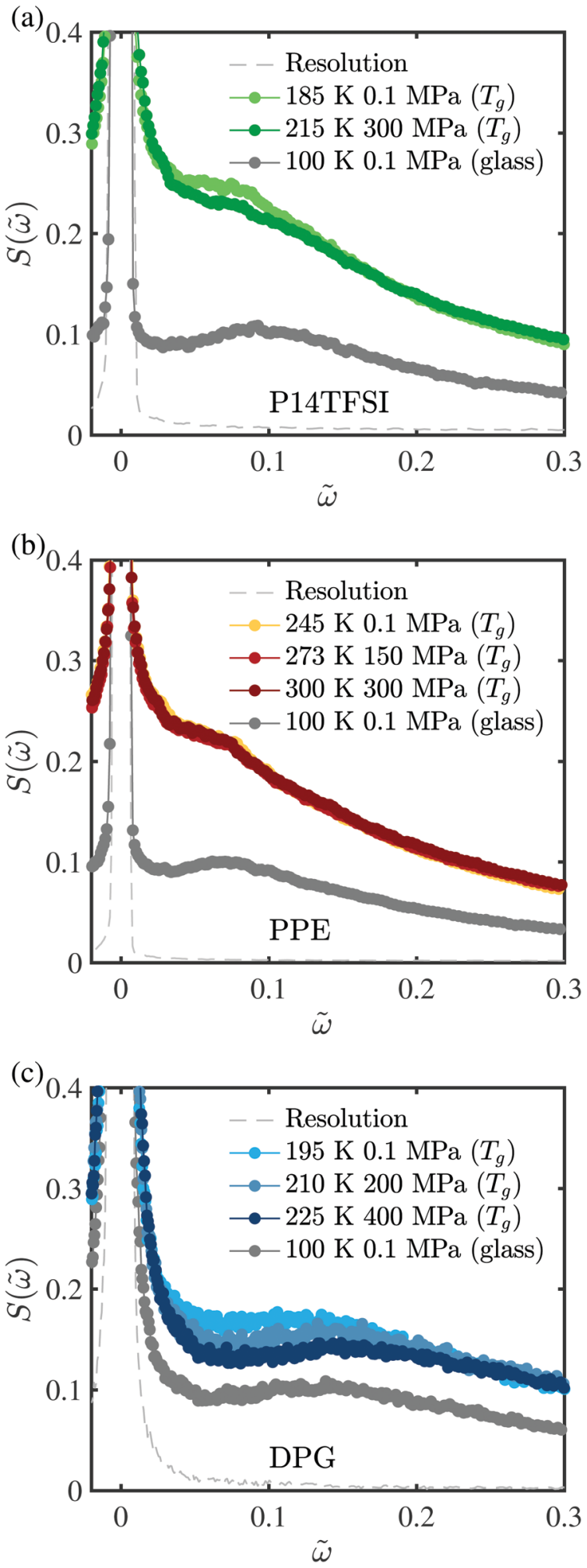

Fig. 7 IN5 spectra on the glass transition isochrone showing the boson peak and fast relaxation. (a) For the ionic liquid Pyr14TFSI studied in this paper. (b) and (c) show data from ref. 19 for comparison. (b) The van der Waals liquid PPE and (c) the hydrogen-bonded DPG. All data are summed over $Q$ in constant $Q$-slices, $Q=1.2$ to $1.8 \AA^{-1}$. relative Boson peak intensity, ${ }^{41,42}$ and it puts the behavior of the ionic liquid in the category with fragile van der Waals liquids, consistent with the findings regarding fragility for similar ionic liquids in ref. 43.

It has previously been shown that the Boson peak and the fast relaxation are invariant along the $T_{\mathrm{g}}(P)$-line, for van der Waals liquids, but not for hydrogen bonded liquids. ${ }^{19}$ Considering the spectra along the $T_{\mathrm{g}}(P)$-line in more detail it is seen that they are not invariant for Pyr14TFSI. On the other hand, keeping in mind that the two state points on the $T_{\mathrm{g}}(P)$-line have been determined just from having the same conductivity, a macroscopic long-time property, it is still a remarkable collapse of the microscopic dynamics including both fast relaxation and Boson peak.

\section{Conclusion}

Density scaling and the consequential collapse of different isolines hold exactly for model liquids governed by inverse power law pair potentials ${ }^{44}$ and can be explained in terms of isomorph theory. ${ }^{45}$ However, the competing van der Waals and Coulomb interactions and the different length scales in the structure of ionic liquids are at odds with these pictures, and in this case there is no a priori reason to believe that density scaling should hold with the same scaling exponent for different dynamic variables. Yet we find density scaling and collapsing isolines of conductivity, microscopic alpha-relaxation, viscosity, self diffusion, macroscopic alpha-relaxation (i.e. the $T_{\mathrm{g}}(P)$-line) and to a large extend microscopic fast dynamics along $T_{\mathrm{g}}(P)$ in the ionic liquid Pyr14TFSI. Thus, the behavior of the ionic liquid is similar to that of a van der Waals liquid, but there are also features that place the ionic liquid as an intermediate between van der Waals liquids and hydrogen bonded liquids: The value of the scaling exponent is intermediate $(\gamma=2.8$ for Pyr14TFSI), and the imperfect collapse of the fast relaxation and Boson peak along the $T_{\mathrm{g}}(P)$-line has reminiscence of the behavior seen for a hydrogen bonded liquid.

Recent simulations of a model ionic liquid have shown that the strength of the Coulomb interactions influence the alpha relaxation time, ${ }^{46}$ and it is also clear from the values of the scaling exponent $\gamma$ that the dynamics of ionic liquids are significantly different from van der Waals liquids. Given that Coulomb interactions are of higher energy than hydrogen bonds, it is nevertheless surprising to find the ionic liquids in an intermediate position - in fact leaning towards the van der Waals behavior. The molecular structure factor peak, scales with the same variable $\Gamma=\rho^{\gamma} / T$, with exponent $\gamma=2.8$, as the dynamics while the charge ordering peak does not obey this scaling. The molecular peak is a measure of nearest neighbor distance and it is the structural feature ionic liquids and van der Waals liquids have in common. Density scaling of the molecular peak therefore supports the picture that the van der Waals interactions and the short range order play the main role for the dynamics, while the Coulomb interactions and the charge ordering play a much less significant role. This picture could be different in ionic liquids where the cation is 
more bulky e.g. with longer alkyl-chains. In these liquids there is yet another level of ordering, ${ }^{17,18}$ which affects the dynamics at ambient pressure. ${ }^{47,48}$

The conductivity and density data presented in this paper can be obtained from the "Glass and Time" data repository http://glass.ruc.dk/data/.

\section{Conflicts of interest}

There are no conflicts to declare.

\section{Acknowledgements}

We would like to acknowledge Dr. Daniel Rauber and Prof. Rolf Hempelmann from Transfercenter Sustainable Electrochemistry, Saarland Universityand KIST Europe for the synthesis of the partly deuterated samples which were used in the neutron experiments. Jesper S. Hansen and Jeppe C. Dyre are thanked for carefully reading and commenting the manuscript. This work was funded by Swedish Research Council (grant 349-2013-605), the Swedish Foundation for Strategic Research (SSF) within the Swedish national graduate school in neutron scattering (SwedNess) and Research Fund Denmark.

\section{References}

1 M. D. Ediger and P. Harrowell, J. Chem. Phys., 2012, 137, 080901.

2 K. Niss and T. Hecksher, J. Chem. Phys., 2018, 149, 230901.

3 A. Tölle, H. Schober, J. Wuttke, O. G. Randl and F. Fujara, Phys. Rev. Lett., 1998, 80, 2374-2377.

4 C. Alba-Simionesco, A. Cailliaux, A. Alegría and G. Tarjus, Europhys. Lett., 2004, 68, 58-64.

5 R. Casalini and C. M. Roland, Phys. Rev. E: Stat., Nonlinear, Soft Matter Phys., 2004, 69, 062501.

6 C. M. Roland, S. Hensel-Bielowka, M. Paluch and R. Casalini, Rep. Prog. Phys., 2005, 68, 1405-1478.

7 A. Triolo, O. Russina, B. Fazio, G. B. Appetecchi, M. Carewska and S. Passerini, J. Chem. Phys., 2009, 130, 164521.

8 C. M. Roland, S. Bair and R. Casalini, J. Chem. Phys., 2006, 125, 124508.

9 A. S. Pensado, A. A. H. Pádua, M. J. P. Comuñas and J. Fernández, J. Phys. Chem. B, 2008, 112, 5563-5574.

10 E. R. López, A. S. Pensado, M. J. Comuñas, A. A. Pádua, J. Fernández and K. R. Harris, J. Chem. Phys., 2011, 134, 144507.

11 K. R. Harris and M. Kanakubo, Phys. Chem. Chem. Phys., 2015, 17, 23977-23993.

12 M. Paluch, S. Haracz, A. Grzybowski, M. Mierzwa, J. Pionteck, A. Rivera-Calzada and C. Leon, J. Phys. Chem. Lett., 2010, 1, 987-992.

13 M. Paluch, E. Masiewicz, A. Grzybowski, S. Pawlus, J. Pionteck and Z. Wojnarowska, J. Chem. Phys., 2014, 141, 134507.
14 M. Paluch, Z. Wojnarowska, P. Goodrich, J. Jacquemin, J. Pionteck and S. Hensel-Bielowka, Soft Matter, 2015, 11, 6520-6526.

15 Z. Wojnarowska, M. Musiał, M. Dzida and M. Paluch, Phys. Rev. Lett., 2019, 123, 125702.

16 S. Cheng, M. Musial, Z. Wojnarowska, A. Holt, C. M. Roland, E. Drockenmuller and M. Paluch, J. Phys. Chem. B, 2020, 124, 1240-1244.

17 C. S. Santos, N. S. Murthy, G. A. Baker and E. W. Castner, J. Chem. Phys., 2011, 134, 121101.

18 J. C. Araque, J. J. Hettige and C. J. Margulis, J. Phys. Chem. B, 2015, 119, 12727-12740.

19 H. W. Hansen, A. Sanz, K. Adrjanowicz, B. Frick and K. Niss, Nat. Commun., 2018, 9, 518.

20 H. W. Hansen, B. Frick, S. Capaccioli, A. Sanz and K. Niss, J. Chem. Phys., 2018, 149, 214503.

21 DOI to ILL data from IN16B: 10.5291/ILL-DATA.6-05-993 and IN5 : 10.5291/ILL-DATA.LTP-6-7.

22 A. Sanz, H. W. Hansen, B. Jakobsen, I. H. Pedersen, S. Capaccioli, K. Adrjanowicz, M. Paluch, J. Gonthier, B. Frick and E. Lelièvre-Berna, et al., Rev. Sci. Instrum., 2018, 89, 023904.

23 B. Frick, J. Combet and L. van Eijck, Nucl. Instrum. Methods Phys. Res., Sect. A, 2012, 669, 7-13.

24 I. Laue-Langevin, LAMP, the Large Array Manipulation Program., http://www.ill.eu/data_treat/lamp/the-lamp-book/.

25 N. J. Brooks, B. L. L. E. Gauthe, N. J. Terrill, S. E. Rogers, R. H. Templer, O. Ces and J. M. Seddon, Rev. Sci. Instrum., 2010, 81, 064103.

26 M. Basham, J. Filik, M. T. Wharmby, P. C. Y. Chang, B. El Kassaby, M. Gerring, J. Aishima, K. Levik, B. C. A. Pulford, I. Sikharulidze, D. Sneddon, M. Webber, S. S. Dhesi, F. Maccherozzi, O. Svensson, S. Brockhauser, G. Náray and A. W. Ashton, J. Synchrotron Radiat., 2015, 22, 853-858.

27 K. R. Harris, L. A. Woolf, M. Kanakubo and T. Rüther, J. Chem. Eng. Data, 2011, 56, 4672-4685.

28 H. Tokuda, K. Ishii, M. A. B. H. Susan, S. Tsuzuki, K. Hayamizu and M. Watanabe, J. Phys. Chem. B, 2006, 110, 2833-2839.

29 A. Martinelli, A. Matic, P. Jacobsson, L. Börjesson, A. Fernicola and B. Scrosati, J. Phys. Chem. B, 2009, 113, 11247-11251.

30 T. B. Schrøder, U. R. Pedersen, N. P. Bailey, S. Toxvaerd and J. C. Dyre, Phys. Rev. E: Stat., Nonlinear, Soft Matter Phys., 2009, 80, 041502.

31 D. Gundermann, U. Pedersen, T. Hecksher, N. P. Bailey, B. Jakobsen, T. Christensen, N. B. Olsen, T. B. Schrøder, D. Fragiadakis, R. Casalini, C. M. Roland, J. Dyre and K. Niss, Nat. Phys., 2011, 7, 816-821.

32 T. A. Lima, L. F. Faria, V. H. Paschoal and M. C. Ribeiro, J. Mol. Struct., 2019, 1183, 149-156.

33 Z. Wojnarowska and M. Paluch, in Dielecitric Properties of Ionic Liquids, ed. M. Paluch, High-Pressure Dielectric Spectroscopy for Studying the Charge Transfer in Ionic Liquids and Solids, Springer, 2016, ch. 4, pp. 73-114.

34 Z. Wojnarowska, L. Tajber and M. Paluch, J. Phys. Chem. B, 2019, 123, 1156-1160. 
35 K. Adrjanowicz, J. Pionteck and M. Paluch, RSC Adv., 2016, 6, 49370-49375.

36 F. Puosi, O. Chulkin, S. Bernini, S. Capaccioli and D. Leporini, J. Chem. Phys., 2016, 145, 234904.

37 M. Romanini, M. Barrio, R. Macovez, M. D. Ruiz-Martin, S. Capaccioli and J. L. Tamarit, Sci. Rep., 2017, 7, 1346.

38 H. W. Hansen, B. Frick, S. Capaccioli, A. Sanz and K. Niss, J. Chem. Phys., 2018, 149, 214503.

39 B. Frick and D. Richter, Science, 1995, 267, 1939-1945.

40 A. I. Chumakov, G. Monaco, A. Monaco, W. A. Crichton, A. Bosak, R. Rüffer, A. Meyer, F. Kargl, L. Comez, D. Fioretto, H. Giefers, S. Roitsch, G. Wortmann, M. H. Manghnani, A. Hushur, Q. Williams, J. Balogh, K. Parliński, P. Jochym and P. Piekarz, Phys. Rev. Lett., 2011, 106, 225501.
41 A. P. Sokolov, E. Rössler, A. Kisliuk and D. Quitmann, Phys. Rev. Lett., 1993, 71, 2062.

42 K. Niss and C. Alba-Simionesco, Phys. Rev. B: Condens. Matter Mater. Phys., 2006, 74, 024205.

43 P. Sippel, P. Lunkenheimer, S. Krohns, E. Thoms and A. Loidl, Sci. Rep., 2015, 5, 13922.

44 U. R. Pedersen, N. Gnan, N. P. Bailey, T. B. Schrøder and J. C. Dyre, J. Non-Cryst. Solids, 2011, 357, 320-328.

45 J. C. Dyre, J. Phys. Chem. B, 2014, 118, 10007-10024.

46 T. Pal and M. Vogel, J. Chem. Phys., 2019, 150, 124501.

47 J. Pitawala, A. Matic, A. Martinelli, P. Jacobsson, V. Koch and F. Croce, J. Phys. Chem. B, 2009, 113, 10607-10610.

48 M. Becher, E. Steinrücken and M. Vogel, J. Chem. Phys., 2019, 151, 194503. 